\title{
Le Bien contre le Mal contre Claus
}

\author{
Le film Le lion des Flandres (1984) et le nationalisme \\ flamand
}

Gertjan Willems ${ }^{1}$

\begin{abstract}
[Résumé] Cet article analyse le film Le Lion des Flandres (Hugo Claus, 1984), en se basant sur une analyse de textes et sur l'exploitation d'archives. II se concentre sur les relations complexes que ce film entretient avec la question nationale belge et flamande. Cette coproduction flamande et néerlandaise (également adaptée en série télévisée en 1985) est une adaptation du roman historique romantique du même nom d'Hendrik Conscience, publié en 1838, une œuvre marquante dans l'histoire culturelle et symbolique du Mouvement flamand. Malgré diverses difficultés liées au caractère nationalise flamand de l'œuvre de Conscience, les producteurs (dont le ministère flamand de la Culture et la télévision publique de la Communauté flamande) voulaient que le film soit le plus fidèle possible au roman de Conscience. Il en a résulté une production ouvertement romantique et nationaliste flamande et ce, en dépit de quelques contrepoints introduits par le directeur de production Hugo Claus ; un personnage controversé et critiqué mais néanmoins reconnu comme étant extrêmement rigoureux. Bien que Le Lion ait été la production belge néerlandophone la plus coûteuse, le film s'est avéré un échec critique et commercial sans précédent.

Mots-clés : Nationalisme / cinéma belge / Hugo Claus / Le lion des Flandres / Hendrik Conscience
\end{abstract}

[Abstract] By means of a textual film analysis and original archival research, this article analyses the film Le Lion des Flandres (Hugo Claus, 1984) and its complex relations with the Flemish and Belgian national question. This Flemish-Dutch co-production (in 1985 also released as a television serial) was an adaptation of Hendrik Conscience's romantic historical novel from 1838 by the same name, a landmark within the cultural and symbolic history of the Flemish Movement. Despite various difficulties concerning the Flemish-nationalist sensitivities of the project, the producers (including the ministry and the public broadcaster of the Flemish Community) wanted the film to be as faithful as possible to Conscience's novel. This largely resulted in an overtly romantic and Flemish-nationalist production, in spite of some counterpoints introduced by the controversial and critical but heavily disciplined director Hugo Claus. Although Le Lion was the most expensive production in the Dutch-language film history, it turned out to be an unprecedented critical and commercial failure.

Keywords: Nationalism / Belgian cinema / Hugo Claus / Le lion des Flandres / Hendrik Conscience

1 Université de Gand. 


\section{Introduction}

Pour commémorer le centième anniversaire de la mort du célèbre écrivain du XIXe siècle, Henri Conscience, une série d'expositions, de publications, de représentations théâtrales et autres manifestations a eu lieu en 1983. Dans ce contexte, l'adaptation audiovisuelle du roman de Conscience de 1838 Le Lion des Flandres ou la Bataille des Eperons d'Or (De leeuw van Vlaanderen of de slag der gulden sporen) par Hugo Claus a été pensée comme une épopée médiévale spectaculaire qui tiendrait lieu de clou du spectacle des commémorations. Offrant un récit romancé de la victoire flamande sur les Français lors de la bataille des éperons d'or en 1302, le roman de Conscience est sans doute l'œuvre littéraire la plus importante du répertoire symbolique et mythologique du Mouvement flamand (l'ensemble des personnes et des associations qui luttent pour l'émancipation flamande) au XIXe et XXe siècle. Le coût de l'adaptation audiovisuelle de 1984 s'élève à 80 millions de francs belges (19,8 millions d'euros), ce qui fait de ce film le plus onéreux produit aux Pays-Bas à l'époque. En dépit du budget alloué, Le lion n'a pas réussi à toucher un large public. Il est devenu l'un des échecs les plus retentissants dans l'histoire du cinéma et de la télévision flamande. Dans un contexte de troubles communautaires et d'émancipation culturelle flamande, une grande partie de la controverse entourant la production a été alimentée par les associations flamandes nationalistes. Dans cette étude, j'examine la manière dont le film, sa production et son accueil se situent dans un contexte de nationalisme flamand. Cet article se veut donc une illustration de la complexité de la façon dont un média peut servir à donner corps à une identité et comment il peut servir des fins nationalistes. Il se base sur des données d'archives du département du film « les dossiers d'archives des médias et du cinéma 2004 » (DAMC 2004) des Archives de l'État belge à Beveren (AEB) ainsi que sur des enregistrements « les dossiers du secrétariat de l'institut »(DSI) de la Vlaamse Radio-en Televisieomroeporganisatie (VRT), à Bruxelles² ${ }^{2}$ Dans une moindre mesure, d'autres archives ont également été consultées, comme celles du Centre de Recherche et de Documentation Hugo Claus à Anvers, celles du Sénat belge et du Parlement flamand. J'utilise ces ressources pour reconstruire et analyser l'histoire de la production du film Le Lion des Flandres. Je prête une attention particulière aux relations de pouvoir entre les différents agents impliqués dans la réalisation du film et la façon dont ils ont tenté d'imposer leur point de vue. Le résultat de cette négociation, le film final, est traité dans une perspective textuelle. Il permet une meilleure compréhension de la façon dont Le Lion des Flandres a été reçu. Pour cette analyse, 92 articles de journaux et de magazines (dont 52 critiques du film) ont été dépouillés. Ceux-

2 Les abréviations suivantes seront désormais utilisées : AEB, DAMC 2004, VRT et DSI. Toutes les citations ont été traduites du néerlandais par l'auteur. 
ci sont disponibles à la Cinémathèque royale (Bruxelles), au Centre de Documentation pour la Presse cinématographique (Bruxelles), et dans les dossiers du Cinema Zuid (Anvers).

\section{Le Lion de Conscience}

En 1838, Conscience publie Le Lion des Flandres, un roman évoquant une bataille connue sous le nom de bataille des éperons d'or. Ces événements historiques datant de 1302 concernent un conflit féodal complexe entre le comte de Flandre (le territoire du comté médiéval ne correspond que partiellement à la Flandre actuelle) et le roi de France (le royaume de France correspond largement à la France actuelle, mais est un peu plus petit). Divers intérêts politiques et sociaux étaient en jeu mais les motivations nationalistes (connectées à des sentiments nationaux ou à une identité flamande) n'existaient pas à l'époque (Lambert, 2000). Cependant, Conscience n'a pas eu pour objectif précis d'écrire un roman historique (Wauters, 2002). Au lieu de cela, Conscience (en tant que partisan du Mouvement flamand qui luttait pour la reconnaissance linguistique et culturelle de la Flandre, région néerlandophone au nord de la Belgique) désirait narrer une histoire romantique dans le but de créer une prise de conscience flamande et un esprit de nation. Le roman revient sur cet événement historique en le présentant sous la forme d'une lutte entre le bien (les Flamands) et le mal (les Français). Dans le prologue et l'épilogue, Conscience $(1838,190)$ parle directement au lecteur pour exacerber son sentiment d'appartenance nationaliste : "Toi, Flamand, qui as lu ce livre, considère par les faits glorieux qu'il relate ce que la Flandre était jadis, ce qu'elle est maintenant et ce qu'elle redeviendra si tu oublies les exemples sacrés de tes aïeux!». De la même manière, dans le reste du livre, Conscience fait allusion au thème romantique de réveil national. Cependant, le roman de Conscience ne peut être compris que dans le contexte de la diversité nationale qui caractérise la Belgique depuis sa formation en 1830 (avec des sentiments nationalistes belges et flamands). La volonté de Conscience de voir les Flamands s'émanciper n'est pas en contradiction directe avec un certain patriotisme belge.

$\mathrm{Au}$ XIXe siècle et au début du XXe siècle, Le lion devint un succès populaire et un symbole culturel pour le Mouvement flamand en développement. Parallèlement à sa notoriété grandissante, le roman prit de plus en plus un aspect symbolique, mais au sein de la culture flamande exclusivement (Tollebeek, 2002), et non au sein de la culture belge. Avec les réformes de l'État belge adoptées au cours de la seconde moitié du $\mathrm{XXe}$ siècle, les conditions sociales, culturelles, politiques et institutionnelles de la Flandre changèrent radicalement. L'État belge unitaire, s'étant transformé en un État fédéral, la Flandre acquit plus d'autonomie à différents niveaux. Cette émancipation flamande élargie provoqua le déclin de la nécessité des histoires héroïques à la gloire des Flamands et de leur passé. Le Lion, devenu un mythe profondément ancré dans les esprits, ainsi que le lieu de la bataille des éperons d'or, devenu l'un des lieux de mé- 
moire les plus forts de Flandre (Nora, 1984), conservèrent cependant leur place dans la mémoire collective.

Pour le centième anniversaire de la mort de l'écrivain, en 1983, une série d'expositions, de publications, de représentations théâtrales et d'autres manifestations eut lieu en l'honneur de Conscience. Le prestigieux film et la série télévisée Le Lion des Flandres furent des éléments-clés de cette commémoration. Le projet fut développé entre 1977 et 1984, dans un contexte marqué par des troubles socio-politiques au sein du Mouvement flamand. Les manifestations flamandes contre le pacte d'Egmont (1977) qui prévoyait une réforme de l'État ont non seulement fait chuter le gouvernement Tindemans II, mais ont également fait apparaître un détachement de l'aile radicale de la Volksunie (un parti politique nationaliste flamand qui faisait partie du gouvernement). Ce détachement prit le nom de "Vlaams Blok». Après la deuxième réforme de l'État en 1980, qui a conduit à une division définitive de la Belgique - les communautés et régions disposant désormais de leur propre pouvoir exécutif -, le développement du Mouvement flamand et plusieurs problèmes communautaires n'ont eu de cesse de causer des tensions en Belgique. Ce contexte fit de l'adaptation audiovisuelle du roman de Conscience - la «bible du nationalisme flamand» - un projet sensible.

\section{La production du Lion}

Vers 1977, le producteur belge Jean Van Raemdonck (souvent mieux connu sous le nom de Jan) demanda à Claus d'écrire un scénario pour une série télévisée basée sur le roman de Conscience, Le Lion. En tant que PDG de Kunst en Kino, à l'époque l'une des plus grandes sociétés de production en Belgique (Willems, 2014), Van Raemdonck voulait produire un spectacle sur le thème des chevaliers, des châteaux et des batailles en Flandre. Mais il manquait d'expérience dans ce genre filmique et le marché intérieur belge, peu intéressé par ce genre de production, rendait la collecte de fonds difficile. Van Raemdonck, espérait cependant que l'année 1983, dédiée à Conscience, lui permettrait de trouver le financement nécessaire pour réaliser son projet.

Le premier partenaire à rejoindre Kunst en Kino fut le diffuseur public flamand BRT. En plus de quelques sponsors secondaires, le diffuseur public néerlandais KRO (Katholieke Radio Omroep) fut également contacté pour coproduire Le Lion. En octobre 1980, Van Raemdonck sollicita une demande de financement auprès du Département du Film du ministère de la Communauté flamande. La Commission du Film du ministère émit un avis négatif sur le projet ${ }^{3}$. La Secrétaire d'État à la Culture - Rika De Backer, du parti politique démocrate-chrétien (CVP, Christelijke Volkspartij) - dé-

3 AEB, DAMC 2004, dossier n 18, Rapport sur la Réunion de la Commission, 6 février 1981. 
cida cependant d'ignorer l'avis de la Commission et octroya la moitié du budget annuel alloué au cinéma à ce projet : 25 millions de francs belges (620.000 euros). ${ }^{4}$. Un tel écart par rapport à l'avis de la Commission était inhabituel et celle-ci exprima son mécontentement dans une note spéciale adressée au nouveau ministre de la Culture, Karel Poma (du parti libéral PVV, Partij voor de Vrijheid), qui remplaça De Backer à la fin de l'année 1981. La Commission fit valoir que l'expérience et les moyens pour produire un tel film historique manquaient aux Pays-Bas. En outre, elle déclara que « de nos jours, l'historiographie héroïque telle qu'elle est pratiquée dans Le lion, n'est plus justifiée et se moque de la vérité historique ${ }^{5}$ ». La Commission du Film était donc fortement opposée à une représentation romantique de l'histoire telle qu'elle était évoquée par Conscience, mais ni De Backer ni Poma ne répondirent à ces préoccupations. Plus tard cependant, la presse repris précisément ces deux arguments - l'insuffisance des moyens et des savoir-faire et le caractère nationaliste romantique du film - pour critiquer Le lion.

Malgré les protestations de la Commission, Kunst en Kino, la BRT, la KRO, et le ministère flamand de la Culture signèrent l'accord de production le 17 mars 1982. Cependant, au sein du conseil d'administration de la BRT, nommé politiquement, aucun consensus ne fut dégagé, concernant l'attrait du film Le Lion. Les représentants CVP, ainsi que l'Administrateur général de la BRT, Paul Vandenbussche (également associé au CVP), étaient en faveur de la production. Ce soutien est quelque peu surprenant étant donné que, dans les années 1980, le drame historique a commencé à perdre beaucoup de sa popularité, en faveur de la fiction contemporaine, jugée plus proche de la réalité quotidienne des spectateurs flamands. Néanmoins, à cette époque, la BRT investit encore davantage dans le drame historique que précédemment. Alexander Dhoest $(2002,261)$ cherche l'explication dans le fait que la formation de la nation flamande faisait partie intégrante de l'agenda social de l'époque, et que la Flandre voulait se présenter culturellement forte, notamment sur le plan de la fiction. Cette perspective s'inscrit dans le rôle plus large qu'a eu la BRT dans la construction d'une identité flamande. La BRT avait pour habitude d'accorder une attention considérable aux épisodes célèbres et aux glorieuses figures de l'histoire flamande (Grégoire, 2006), telles celles que représentait Le Lion.

L'opposition au projet provint principalement des représentants libéraux Armand Beyens et Adriaan Verhulst (un éminent flamingant). Antoon Van Overstraeten, représentant du parti politique pro-flamand Volksunie, fut également l'un d'eux. Les membres du conseil les plus pro-flamands s'opposèrent donc à l'adaptation de l'œuvre littéraire la plus importante du Mouvement flamand. Les difficultés financières et de 
production furent mises en avant, mais la principale raison de cette apparente contradiction trouve vraisemblablement sa source dans l'évolution de l'idéologie nationaliste flamande, qui prit de plus en plus de distance avec les aspects romantiques et mythiques du passé. Il se peut également que cette opposition soit liée à l'évolution plus générale concernant la déconstruction des mythes nationaux. En tant qu'historien, Verhulst critiqua en effet régulièrement le manque de précision historique du scénario. Les autres membres du conseil d'administration firent néanmoins valoir que le but n'avait jamais été de présenter une représentation historique des événements: "L'objectif est l'adaptation d'un sujet que Conscience a transformé en une épopée nationale ${ }^{6} »$. Cela souligne le fait que les partisans de l'adaptation étaient pleinement conscients du caractère nationaliste romantique du projet. Dans le même temps, l'accent mis sur le roman de Conscience plutôt que sur les événements historiques de 1302, peut également être considéré comme une stratégie visant à dépolitiser la représentation des événements historiques. Il est possible que le raisonnement était que si le film était traité comme une fiction, les producteurs ne pouvaient être soupçonnés de poursuivre des motivations idéologiques concernant la représentation spécifique du passé. Cependant, on peut objecter ici que ce raisonnement n'a pas pris en considération le fait que la fiction peut aussi être interprétée en termes politiques et idéologiques. Après des mois de débats houleux, plusieurs opposants au projet se laissèrent finalement convaincre lorsqu'une version plus modeste du projet fut proposée, à laquelle la BRT participa dans une moindre mesure?

L'un des points sensibles du processus de production fut la nomination de Claus en tant que réalisateur du film. La KRO avait suggéré Claus - la figure littéraire d'aprèsguerre la plus importante de Flandre - car il était le seul réalisateur flamand à être également un personnage public aux Pays-Bas. Cependant, plusieurs personnes au sein de la BRT désapprouvèrent ce choix ${ }^{8}$, parmi lesquelles les membres les plus proflamands du conseil d'administration, Verhulst et Van Overstraeten. La réputation de Claus explique la controverse autour du choix de sa personne en tant que réalisateur. Depuis son premier roman en 1950, Claus s'est en effet révélé être l'enfant terrible de la culture flamande, aux yeux du grand public (Absillis, 2005). Claus a toujours eu une relation ambiguë avec la Flandre, en la critiquant et en ayant en même temps une forte attraction pour elle. Mais il se fit surtout connaître pour son attitude critique à l'égard du nationalisme flamand.

6 VRT, DSI 5803, dossier n 335, Rapport sur la Réunion du conseil d'administration n 32, 3 mai 1982.

7 VRT, DSI 5803, dossier n 335, Rapport sur la Réunion du conseil d'administration n 34, 7 juin 1982.

8 AEB, DAMC 2004, dossier n² 249-253, Rapport sur la Réunion concernant la nomination d'un réalisateur, 14 janvier 1982. 
Néanmoins, Claus accepta la proposition. Plusieurs raisons ont probablement motivé son choix. D'une part, il avait toujours voulu toucher un public aussi vaste que possible, notamment avec ses créations cinématographiques (Jacobs, Landuyt, Lembrechts, Wildemeersch, 2004). À cet égard, Le Lion apparut comme une superproduction flamande prometteuse. Dans le même temps, Claus argua qu'il souhaitait réaliser une grande épopée, un vrai film de divertissement médiéval et que la seule possibilité pour qu'un tel projet voie le jour en Flandre était d'adapter le roman de Conscience (Sojcher 1999, 297). En outre, Claus pensait qu'il serait capable de modeler le projet à sa guise, tout comme il l'avait fait pour l'adaptation théâtrale de la parodie du roman Goudland de Conscience, en 1966.

Néanmoins, les producteurs souhaitaient une adaptation "fidèle » du roman de Conscience et tentèrent d'empêcher Claus de donner à son adaptation une touche critique ou ironique. Les producteurs établirent un comité de surveillance chargé de l'approbation des plans du projet, de la surveillance, de l'avancement de la production et de l'approbation du montage final ${ }^{9}$. Par conséquent, Claus fut limité dans sa liberté artistique et les producteurs gardèrent le contrôle de la production. Par exemple, lorsque Claus entendit suivre les conseils du consultant historique, le Comité l'avertit « qu'il incombe au réalisateur d'adapter l'œuvre de Conscience, pas de l'améliorer ${ }^{10}$ ».

\section{Le Bien contre le Mal contre Claus}

L'analyse textuelle du film tel qu'il a finalement été produit montre que les producteurs ont largement réussi à imposer leur volonté. Il faut souligner que, dans le cadre scolaire, ce genre de films, basés sur un classique de la littérature et évoquant un passé glorieux, est souvent appréhendé d'un point de vue nationaliste (voir, par exemple, Chapman, 2005; Higson, 2006). L'analyse de la façon dont la culture et l'identité nationale sont représentées et construites prend une place centrale dans les discussions (Anderson, 1983; Hall, 1997). L'adaptation d'un classique de la littérature fournit en outre une dimension supplémentaire, car elle contribue à la construction et à l'entretien d'un patrimoine littéraire.

Tenant compte de ces considérations, j'examine le rapport entre le film et la question nationale. Cette démarche implique évidemment de se pencher sur la question du rapport entre le film et le livre de Conscience. Mais elle nécessite également d'analyser le traitement cinématographique du sujet historique. Tout comme d'autres recherches se sont penchées sur la façon dont le Moyen-Age est évoqué (Aberth, 2003;

9 AEB, DAMC 2004, dossier n² 249-253, Accord de production, 10 juin 1982.

10 AEB, DAMC 2004, dossier n² 249-253, Rapport sur la Réunion du Comité de surveillance, 20 mai 1983. 
Marshall, 2007), cette analyse de filmique entend mieux comprendre le traitement d'un épisode particulier de l'histoire médiévale flamande, au début des années 1980. Car, comme Pierre Sorlin l'indique (1980), un film historique en dit souvent plus sur la période pendant laquelle il a été tourné, que sur la période visée par le film.

Au début du film, le comte de Flandre Guy de Dampierre (né en 1226 et comte de Flandre à partir de 1253 jusqu'à sa mort en 1305) et ses compagnons sont emprisonnés par les Français. Quand le roi de France, Philippe le Bel (né en 1268 et roi de France de 1285 jusqu'à sa mort en 1314) nomme Jacques de Châtillon au poste de nouveau gouverneur de Flandre, la décision terrorise les habitants de la ville de Bruges. Lorsque la famille de Jan Breydel, chef de la corporation des bouchers, est assassinée, le gouverneur rencontre le chef des tisserands, Pieter De Coninck, et l'enjoint à prendre la ville aux Français (évènement appelé les " Matines de Bruges »). Le roi de France, épris de vengeance, rassemble une armée pour l'ultime confrontation du 11 juillet 1302. Avec l'aide de Robert de Béthune, le fils du comte de Flandre, vêtu d'une armure d'or, les Flamands (le peuple du comté de Flandre, dont le territoire ne correspond que partiellement à la Flandre actuelle) gagnent la bataille des éperons d'or. Bien que le roman historique de Conscience soit évidemment plus élaboré dans les détails, les histoires et les personnages, la narration du film correspond en grande partie à celle du roman. Cela implique qu'il existe plusieurs inexactitudes historiques et anachronismes dans le film. Par exemple, contrairement à ce qui est relaté dans le film, ni Breydel ni De Coninck n'ont participé aux événements des " Matines de Bruges », et de Béthune a été emprisonné lors de la bataille des éperons d'or.

Le film suit également le roman de Conscience en créant une dichotomie entre les " gentils Flamands» et les "méchants Français». La construction d'un "nous » (les Flamands) et d'un «autre» (les Français) est très claire. Le premier personnage flamand présenté dans le film est Jan Breydel. Son combat contre les soldats et les chevaliers français pour libérer un paysan symbolise le courage héroïque des Flamands. Dans le même temps, l'idée principale du film est introduite : faibles en apparence, les Flamands parviennent à libérer la Flandre des oppresseurs Français grâce à leurs traits de caractère exceptionnels tels que l'honneur et l'héroïsme. En dépit du fait que la Flandre médiévale des années 1300 était un tout autre territoire que la Flandre actuelle, l'image du «Flamand»-présenté comme membre d'un peuple ancestral qui survit à travers les siècles - est créée. Le film suggère donc que les Flamands d'aujourd'hui sont directement liés aux Flamands courageux du film.

À l'opposé du brave et honorable Flamand; la décadence et la lâcheté des Français qui ont trahi l'ordre de la chevalerie. Les personnages français personnifient le mal et font régner une véritable terreur aux alentours de Bruges. Leur cruauté est illustrée par le meurtre de la famille innocente de Breydel. La vengeance du Flamand, lors des "Matines de Bruges », est tout aussi impitoyable mais elle est présentée comme dans le roman de Conscience, c'est-à-dire que la violence est considérée comme justifiée (Tollebeek 2004, 335). La «bonté» des Flamands est soulignée par deux oppositions 
simplistes aux «mauvais » Français. La présentation des personnages français et flamands, en tant que couples opposés, pousse le spectateur à relire les événements historiques de 1302 comme une lutte entre le Bien et le Mal. Ainsi, le comte de Flandre est opposé au roi de France, Béthune est opposé à Châtillon et Mathilde de Béthune est opposée à Jeanne Ière de Navarre. S’il est évident que, pour réussir un film grand public, il est presque toujours nécessaire de simplifier certains faits historiques et d'introduire quelques éléments fictifs (Rosenstone, 1995), sur la base de l'analyse précédente, je voudrais argumenter que le fait de réduire les événements historiques de 1302 à une opposition nationaliste entre « le Bien » et « le Mal » va à l'encontre de l'esprit de l'histoire et limite ainsi la valeur historique du film. Bien sûr, cette présentation duale est liée à la fidélité du film vis-à-vis du roman de Conscience, à qui la même critique historique peut être appliquée.

Cependant, à certains égards, le film se distingue du roman. Fidèle à sa réputation de provocateur, Claus a mentionné dans des interviews qu'il voulait se débarrasser du caractère pieux de l'œuvre de Conscience. La volonté de Claus de passer outre l'aspect religieux se manifeste notamment à travers la représentation de la féminité dans le film. L'illustration la plus claire des éléments critiques et ironiques de Claus apparaît à la fin du film, lorsque les Flamands trouvent le costume d'or de l'armure de de Béthune qui a joué un rôle décisif dans la victoire de la bataille des éperons d'or. Ils honorent son armure comme une relique et l'accrochent sur une croix, faite de branches. Le peuple, massé en foule, entame alors un pèlerinage vers la relique tandis que la voix-off narre : «Alors nous nous sommes tous agenouillés et nous avons chanté, nous avons offert fièrement nos prières reconnaissantes au ciel miséricordieux, demeure des saints, demeure de notre chevalier d'or. Que Dieu le bénisse pour l'éternité ». La dévotion exagérée et l'association facile de l'armure sur la croix avec un vulgaire épouvantail, nient le caractère héroïque de l'événement. Les Flamands se mettent à genoux pour un symbole représentant un mort. Cette attitude peut être interprétée comme une critique du romantisme lié au nationalisme flamand. Cependant, de manière générale, de tels éléments critiques et/ou ironiques sont relativement peu nombreux dans le film. Par conséquent, les aspects romantiques et nationalistes de la version de Conscience restent dominants, rendant le film assez fidèle au roman de Conscience et font du film une adaptation fidèle à la vision des producteurs. Tout comme le roman, de façon générale, le film donne donc corps à une identité nationaliste flamande.

\section{L'accueil reçu par Le Lion}

Les producteurs ont fourni beaucoup d'efforts pour commercialiser le film en tant qu'événement cinématographique majeur. Effectivement, le gala de première du film lors du Festival du Film de Bruxelles, qui s'est tenu le 27 janvier 1984, a attiré l'attention des médias. A côté de diverses personnalités politiques et culturelles de premier 
plan, le roi et la reine de Belgique ont assisté à la projection. Pendant les préparatifs de la première, le secrétaire de la reine a tenu à ce qu'un certain nombre de francophones soient invités afin que le spectacle «ne deviennent pas une affaire purement flamande $\mathrm{e}^{11}$ \%. Ces considérations illustrent le contexte social mouvementé qui caractérise la Belgique au moment de la sortie du film.

Dans ce contexte, plusieurs manifestations séparatistes de l'aile radicale du mouvement flamand ont eu lieu. Lors de la première, aucune échauffourée importante n'a été recensée. Cependant, le parti politique flamand d'extrême droite, le Vlaams Blok, a fait circuler des tracts appelant à la dissolution de la Belgique, avec des slogans tels que «België barst», ce qui signifie "que la Belgique éclate» (Butstraen 1984, 30 janvier). Ces actions ont souligné l'importance symbolique que le thème du film a pris au sein du Mouvement flamand. La connotation politique du film était également exacerbée, au sein du Conseil flamand (le précurseur du Parlement flamand), en raison du coût du film ${ }^{12}$ et de l'indignation manifestée par le sénateur francophone (et militant du Mouvement wallon), Jean-Émile Humblet (Rassemblement wallon). Ce dernier s'est notamment exprimé sur l'adaptation du Lion dans le magazine Forum de l'Armée ${ }^{13}$.

Alors que la presse belge francophone exprima clairement ses préoccupations relatives à l'attitude des associations nationalistes flamandes, la presse flamande, davantage préoccupée par la production à grande échelle, ne prêta que peu d'attention à ces mêmes nationalistes, avant la sortie du film. La personnalité du directeur de production, Claus, connu pour sa posture critique, balaye rapidement la possibilité d'une production nationaliste flamande (voir, par exemple, Trappeniers 1984, 29 janvier). Après la première du film, la majorité des critiques flamands ont rédigé un avis négatif sur le film. Le film a été critiqué pour son aspect terne, son incohérence et son amateurisme, illustré notamment par le recours trop peu abondant à des figurants pour les scènes de foule. Pour les journalistes flamands, la question de la relation du film au nationalisme n'était, encore une fois, qu'une préoccupation mineure. Étant donné que le roman de Conscience était si fortement ancré dans la mémoire collective flamande, il est possible que le potentiel nationaliste du film soit passé inaperçu ou presque, dans le chef des spectateurs. Si le sujet était quand même abordé par la presse, celui-ci n'était pas traité en profondeur. La plupart des commentateurs jugèrent que Claus n'avait apparemment pas disposé d'une assez grande latitude pour

11 AEB, DAMC 2004, dossier n² 249-253, Réunion sur la première, 4 janvier 1984.

12 Parlement flamand, Archive documents parlementaires, Bulletin de questions et réponses, Session 1985-1986, le 15 avril 1986, n 6, Question nº 120 de 14 mars 1986 de Mia PanneelsVan Baelen.

13 Sénat belge, Section archivage et l'historiographie, Bulletin de questions et réponses, le 3 Janvier 1984, n 13, Question nº 17 le 6 décembre 1983 de Jean- Émile Humblet. 
s'exprimer. Le journal néerlandophone De Standaard (Butstraen 1984, 3 février) comprit les touches d'ironie dans le film davantage comme une expression d'amateurisme que comme une critique du nationalisme.

Contrairement à leurs collègues flamands, les critiques négatives rédigées par les Belges francophones se sont principalement basées sur une interprétation politique et idéologique du film. Le journal francophone La Libre Belgique (Anonyme, 1984, 28 janvier) titrait par exemple «Un Film néerlandophone violemment anti-français ». Le journal francophone La Dernière Heure (cité dans Devos 1984) associait le film aux relations communautaires tendues de l'époque et parlait d' "un film capable d'enflammer les esprits ». En dépit du fait que le film met en scène des «Français » et non des Belges, plusieurs journalistes craignaient que le film n'incite les jeunes flamands à développer des sentiments de haine et de violence à l'égard de la Wallonie. André Ceuterick (1984, 3 février), le critique du journal francophone Le Peuple, n'a pas manqué de souligner le soutien que le ministère de la Communauté flamande a apporté à ce film, qualifié de nationaliste flamand et jugé dangereux : "Si les adolescents flamands (...) se passionnent pour les exploits du Lion des Flandres (...), on les verra bientôt défiler dans nos rues au pas cadencé et répandre la terreur dans nos cités au cri de 'In de naam van de Leeuw'. Et nos grand-mères verront se raviver des souvenirs que le temps n'a pu effacer. Un programme très généreusement coproduit par le Ministère de la Communauté Flamande !».

La presse flamande fut quelque peu surprise par ces lectures nationalistes. Il y eut aussi une infime mais bien distincte partie des nationalistes flamands (incarnée notamment par le magazine néerlandophone 't Pallieterken) qui critiqua le film pour des raisons radicalement opposées à celles de la presse francophone: elle dénonça le manque de respect pour le roman de Conscience. Ces nationalistes soulignaient que les quelques éléments ironiques présents dans le film tournaient en ridicule la culture et l'idéologie flamande (voir par exemple D’Haese 1984, 23 février). Le film a donc été fortement critiqué, par divers acteurs. En conséquence, Claus s'empressa de prendre ses distances avec son film.

\section{Conclusion}

Le producteur Van Raemdonck et la BRT voulaient produire un film prestigieux afin de commémorer Conscience et son roman de 1838 Le Lion des Flandres, un symbole du Mouvement flamand. Bien que plusieurs organismes de financement aient été impliqués dans la production du film, des objections ont été soulevées par la Commission du Film flamand qui jugeait le projet de film ouvertement nationaliste. Les producteurs voulaient en effet que le film soit le plus fidèle possible au roman de Conscience, indéniablement nationaliste. Malgré quelques contrepoints ironiques et critiques introduits par le réalisateur controversé Claus, le film de 1984 est en effet essentiellement romantique et nationaliste flamand. Comme dans le roman de Conscience, la 
représentation sélective et les inexactitudes historiques servent à illustrer la victoire des nationalistes, « les bons Flamands », sur «les mauvais Français». Bien que le film ait été la production la plus coûteuse de l'histoire du cinéma belge, il a été jugé négativement par une grande partie des commentateurs et s'est avéré être un échec critique et commercial sans précédent. Cette étude de cas du Lion contribue non seulement à retracer l'histoire du récent nationalisme culturel en Flandre et en Belgique, mais illustre également la complexité et les ambiguïtés de la production et de la réception d'un discours nationaliste.

\section{Bibliographie}

ABERTH J. (2003) : A knight at the movies. Medieval history on film, New York: Routledge.

Absillis K. (2005): " Je faam is, geloof ik, definitief gevestigd : Hugo Claus' opmerkelijke intrede in de Vlaamse letteren », in WiLDEMEERSCH G., éd. (2005) : Het teken van de ram 4. Amsterdam: De Bezige Bij.

ANDERSON B. (1983): Imagined communities : reflections on the origin and spread of nationalism, New York: Verso.

ANONYme (1984, 28 janvier) : «De leeuw van Vlaanderen. Un film néerlandophone violemment antifrançais ». In La Libre Belgique.

Butstraen R. (1984, 30 janvier) : « Donderend applaus voor 'Leeuw' ». In De Standaard.

Butstraen R. (1984, 3 février) : « De vorstinnen van Brugge ». In De Standaard.

Ceuterick A. (1984, 2 février): «Festival de Bruxelles: trois chefs-d'oeuvre pour l'apothéose ». In Le Peuple.

CHAPMAN J. (2005) : Past and present : national identity and the British historical film, London: IB Tauris.

Conscience H. (1838): De leeuw van Vlaenderen of de slag der gulden sporen (Vol. 3), Antwerpen : De Cort.

Devos W. (1984) : « Lettre Flamande ». In Septentrion, nº 2, pp. 84-88.

D’HAESE J. (1984, 23 février) : « De leeuw van Claus ». In 't Pallieterke.

DHOEST A. (2002) : De verbeelde gemeenschap. Vlaamse tv-fictie en de constructie van een nationale identiteit, Leuven : KUL.

GRÉGOIRE J. (2006): Etude de l'histoire à la télévision dans ses rapports avec l'historiographie, la pédagogie et la culture populaire, spécialement en Belgique (19531995). Complicité et dissension d'un couple à la culture disparate, Liège : Université de Liège. 
HaLl S., éd. (1997): Representation : cultural representations and signifying practices, London : Sage.

Higson A. (2006) : " Re-presenting the national past: nostalgia and pastiche in the heritage film », in Friedman L. D., éd. (2006) : (Fires were started : British cinema and Thatcherism. London: Wallflower Press.

JACOBS K., LANDUYT K., LEMBRECHTS K., WiLDEMEERSCH G. (2004) : Hugo Claus : voor twaalf lezers en een snurkende recensent, Rijswijk : Elmar.

LAMBERT V. (2000): « De Guldensporenslag van Fait-Divers tot Ankerpunt van de Vlaamse Identiteit (1302-1838) : De Natievormende Functionaliteit van HistorioGrafische Mythen ", Bijdragen en Mededelingen betreffende de Geschiedenis der Nederlanden, vol. 115, $n^{\circ}$ 3, p. 365-391.

MARShall D. W., ed. (2007) : Mass market medieval. Essays on the Middle Ages in popular culture, Jefferson: McFarland.

NoRa P. (1984) : Les lieux de mémoire, Paris : Gallimard.

ROSENSTONE R. A. (1995): Visions of the past : the challenge of film to our idea of history, Cambridge : Harvard UP.

SOJCHER F. (1999) : La kermesse héroïque du cinéma belge: le miroir déformant des identités culturelles (1965-1988) (Vol. 2), Paris : l'Harmattan.

SORLIN P. (1980) : The film in history. Restaging the past, Totowa: Blackwell Oxford.

TollebeEK J. (2002) : « De cultus van 1302 : twee eeuwen herinneringen », in R. van Caenegem, ed. (2002) : 1302 : feiten en mythen van de Guldensporenslag. Antwerpen: Mercatorfonds.

Tollebeek J. (2004) : « Consciences niemandsland: 'De Leeuw van Vlaenderen', de natie en de geschiedenis », in Delsaerdt P., de Schepper M., ed. (2004): Letters in de boeken: liber amicorum Ludo Simons. Kapellen : Pelckmans.

TRAPPENIERS L. (1984, 29 janvier) : « De Leeuw heeft voor het eerst gebruld ». In Het Laatste Nieuws.

WAUTERS K. (2002): "Literair-historisch essay over De Leeuw van Vlaanderen ", in Conscience, H., Vanhoutte, E, Wauters, K., ed.(2002) : De Leeuw van Vlaanderen of de Slag der Gulden Sporen : tekstkritische editie. Tielt : Lannoo.

Willems G. (2014) : « The producer and Belgian cinema(s) : the case of Jean (and Jan) Van Raemdonck », in SPICER, A., McKennA, A.T., MeIr, C., éd. (2014) : Beyond the bottom-line : the producer in film and television studies. New York : Bloomsbury. 